\title{
Escape - capture bigeminy caused by blocked atrial extrasystoles
}

Sir,

Dubb and Schamroth $(1975,37,890)$ state that three basic mechanisms for escape-capture bigeminy have so far been described. After enumerating these, they present 'a hitherto unrecognized mechanism', viz. blocked atrial extrasystoles. This mechanism has already been described by Weiss (1973).

In Dubb and Schamroth's case the T wave of the sinus beat only appears to be equiphasic since an upright ectopic $\mathbf{P}^{\prime}$ wave is superimposed upon it. This $T$ wave is inverted and the inversion of $T$ in the escape beat is only slightly greater. The $T$ inversion in the escape beat has been labelled 'postextrasystolic'. Post-extrasystolic T wave change is a primary change which occurs in sinus beats following extrasystoles, but not in escape beats following blocked atrial extrasystoles, since in the absence of contraction of almost empty ventricles, the pause is similar to that seen in a block with dropped beats, which are never associated with any $T$ wave change in the following sinus or escape beats. Aberrancy, on the other hand, is quite common in escape beats. The escape beats in this case clearly show aberrant ventricular conduction, leading to an increase in amplitude of QRS in the lead illustrated. This must, of necessity, result in an increase in the $T$ inversion as a secondary $T$ wave change. Hence it is not a postextrasystolic $T$ wave change and is devoid of any pathological significance.

When escape-capture bigeminy results from a basic 3:1 sinoatrial block with escape beats, if the escape beat occurs 'coincidentally with' the first sinus beat of the next ensuing 3:1 sequence as stated by the authors, the sinus beat will not be conducted to the ventricle; in the absence of capture of the ventricle by the sinus beat, there can be no escape-capture bigeminy. In order that escapecapture bigeminy may be possible with 3:1 SA block, the escape beat should not be coincident with but should precede the sinus beat at a sufficient interval, thus permitting the latter to capture the ventricle; or alternatively, the block ratio should be $3: 2$ and not $3: 1$.

\author{
Doctor Jamaluddin, \\ Sir Ganga Ram Hospital, \\ Lahore, \\ Pakistan.
}

\section{Reference}

Weiss, I. W. (1973). Essentials of Heart Rhythm Analysis, p. 209. F. A. Davis, Philadelphia.

This letter was shown to Dr. Dubb and Professor Schamroth who reply as follows:

Sir,

We would like to express our appreciation to Dr. Jamaluddin for his interest in our paper 'Escapecapture bigeminy caused by blocked atrial extrasystoles', and to thank him for drawing our attention to his observation that the mechanism had already been described by Weiss. We were unaware of this, and are still unable to obtain the book.

Dr. Jamaluddin is incorrect in stating that 'the $T$ wave of the sinus beat only appears to be equiphasic since an upright ectopic $\mathrm{P}^{\prime}$ wave is superimposed upon it'. The ectopic $\mathbf{P}^{\prime}$ wave is superimposed on the proximal part of the ST segment and is well clear of the T wave; as is quite evident in the tracing. The $T$ wave is obviously inverted in the escape beat, and thus differs significantly from the equiphasic $T$ wave of the conducted sinus beat. The $T$ wave change of the escape beat is, therefore, a true, so-called 'post-extrasystolic' $T$ wave change. Dr. Jamaluddin is further incorrect in stating that these $T$ wave changes are never associated with block. Indeed, it was Scherf (1944) who originally indicated that the change is not the result of the extrasystole as such, but rather of the pause which it evokes. It may thus be associated with any sudden long pause such as occurs with blocked 
atrial extrasystoles or following the relatively long ventricular cycles in atrial fibrillation.

We do not understand Dr. Jamaluddin's reference to, 'the absence of contraction of almost empty ventricles'. Furthermore, we very much doubt whether the escape beats clearly show aberrant ventricular conduction, as he states. The slight change in amplitude of the QRS complex could be a positional effect resulting from overfilling of the ventricles, and was not evident in other leads. However, even if this change is indeed aberration, it is so slight that it could not account for the real inversion of the associated $T$ wave.

Dr. Jamaluddin is correct in pointing out that with a basic 3:1 sinoatrial block, escape capture bigeminy cannot occur when the escape beat occurs coincidentally with the first sinus beat of the next ensuing
3:1 sequence, as was stated in our text. He correctly points out that the escape beat must precede the next conducted sinus beat. We very much regret this momentary aberration on our part.
A. Dubb,
L. Schamroth, Baragwanath Hospital, P.O. Bertsham, Johannesburg, South Africa.

\section{Reference}

Scherf, D. (1944). Alterations in the form of the T waves with changes in heart rate. American Heart fournal, 28, 332 